\title{
$\gamma$-Cyclodextrin Increases Hydrolysis of Gangliosides by Sialidase from Arthrobacter ureafaciens: Hydrolysis of Gangliosides
}

\author{
Rie Mitsumori, ${ }^{1}$ Tomohisa Kato, ${ }^{1,2}$ and Kenichi Hatanaka ${ }^{3}$ \\ ${ }^{1}$ Japan Chemical Innovation Institute, 1-3-5 Jimbocho, Chiyoda-ku, Tokyo 101-0051, Japan \\ ${ }^{2}$ Kaneka Corporation, 3-2-4, Nakanoshima, Kita-ku, Osaka 530-8288, Japan \\ ${ }^{3}$ Institute of Industrial Science, The University of Tokyo, 4-6-1, Komaba, Meguro-ku, Tokyo 153-8505, Japan \\ Correspondence should be addressed to Kenichi Hatanaka, hatanaka@iis.u-tokyo.ac.jp \\ Received 3 November 2008; Accepted 16 December 2008 \\ Recommended by Chun-Hung Lin
}

Sialidase is a ubiquitous enzyme that catalyzes the hydrolytic removal of terminal sialic acid residues from oligosaccharides in glycolipids and glycoproteins. Ganglioside GM1 has been usually found to be resistant to various sialidases. Arthrobacter ureafaciens sialidase has been reported to remove sialyl residues of ganglioside GM1 in the presence of bile salts. However, bile salts are difficult to be removed, and disturb HPTLC analysis. Using $\gamma$-cyclodextrin $(\gamma$-CD) as a novel additive agent, ganglioside GM1 was efficiently hydrolyzed to asialo-GM1 by $A$. ureafaciens sialidase.

Copyright ( 2009 Rie Mitsumori et al. This is an open access article distributed under the Creative Commons Attribution License, which permits unrestricted use, distribution, and reproduction in any medium, provided the original work is properly cited.

\section{Introduction}

Gangliosides are glycolipids which contain sialic acid residues with $\alpha 2-3, \alpha 2-6$, and $\alpha 2-8$ linkages, and are primarily localized on the outer surface of mammalian cells. The biologic functions of gangliosides are attributed to the acidic carbohydrate chains [1] and have been found to be important for cell growth, differentiation, cell-to-cell interactions, and signal transduction $[2,3]$. The linkages of sialic acid residues are enzymatically hydrolyzed by various sialidases from viruses, bacteria, and mammals $[4,5]$. While sialidase from leech, Macrobdella decora [6, 7], does not cleave the sialic acid residues of GD1b and GT1b to form GM1, the major gangliosides in mammalian brain, GD1a, GD1b, and GT1b are hydrolyzed into GM1 with most commercially available sialidases of bacterial origin, such as Vibrio cholerae and Clostridium perfringens [8, 9]. These bacterial sialidases cleave the external NeuAca2-3Gal in GD1a and GT1b, and NeuAc $\alpha 2-8 \mathrm{NeuAc}$ in GD1b, but not the internal NeuAc $\alpha 2$ $3 \mathrm{Gal}$ due to the steric hindrance by neighboring GalNAc $[6,10,11]$. However, three isoenzymes (L, M1, M2) from Arthrobacter ureafaciens can hydrolyze the internal sialic acid of GM1 under optimal condition with sodium cholate [7]. Recently, it was reported that the C-terminal peptide of isoenzyme M2 is essential to prevent the effect of the neighboring GalNAc and cleave the sialic acid at the internal galactose residue [12]. Recently, it was reported that $y$ cyclodextrin $(\gamma-\mathrm{CD})$ was an efficient supporting material for glycosyltransferase reaction [13]. Therefore, an effect of $\gamma$ $\mathrm{CD}$ on sialidase reaction is interesting.

Recently, it was reported that asialo-GM1 contributes to various diseases such as carcinogenesis, autoimmune disease, or neurologic disease $[14,15]$. It is hoped that understanding of action mechanisms of various asialo gangliosides is useful for medical treatment in future. In this paper, we attempted to prepare asialo gangliosides by using $A$. ureafaciens sialidase and $\gamma$-CD.

\section{Materials and Methods}

2.1. Materials. All organic solvents of analytical grade, ganglioside GM1, crude ganglioside mixture (from rat brain), and A. ureafaciens sialidase (mixture of isoenzymes L, M1, M2 and S) were purchased from Nacalai Tesque, Kyoto, Japan. $\alpha$-cyclodextrin $(\alpha$-CD), $\beta$-cyclodextrin $(\beta$-CD), and $\gamma$-CD were purchased from WAKO, Osaka, Japan. Sep-Pak C18 columns were purchased from Waters, Milford, MA, USA. 


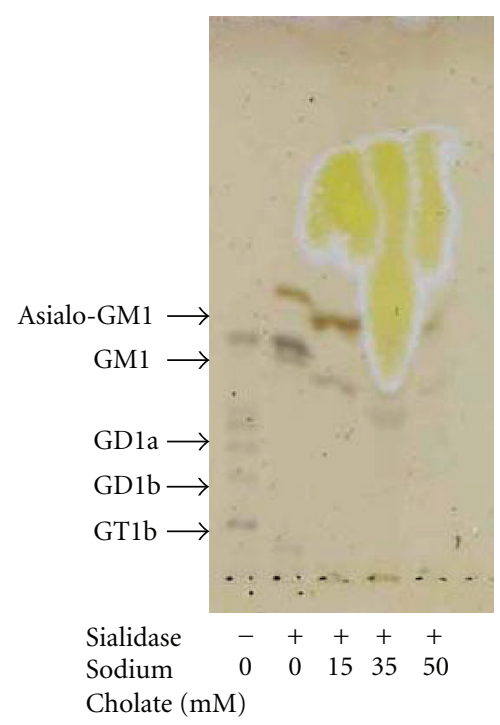

(a)

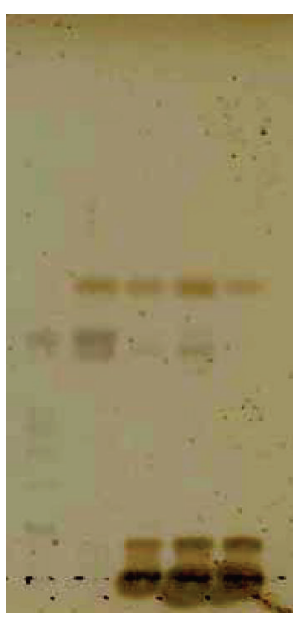

Sialidase $\quad-++++$ $\gamma$-CD (mM) $0015015 \quad 35 \quad 50$

(b)

FIGURE 1: Effect of (a) sodium cholate and (b) $\gamma$-CD on hydrolysis of gangliosides by A. ureafaciens sialidase. $200 \mathrm{mU}$ of sialidase was added $(+)$ or not added $(-)$ to the reaction solution. Samples purified by using Sep-Pak C-18 cartridge were analyzed by HPTLC with chloroform/methanol/0.25\% aqueous $\mathrm{KCl}(5: 4: 1, \mathrm{v} / \mathrm{v})$ as developing solvent. The HPTLC plates were sprayed with resorcinol reagent and heated at $120^{\circ} \mathrm{C}$ to detect the separated glycolipids.

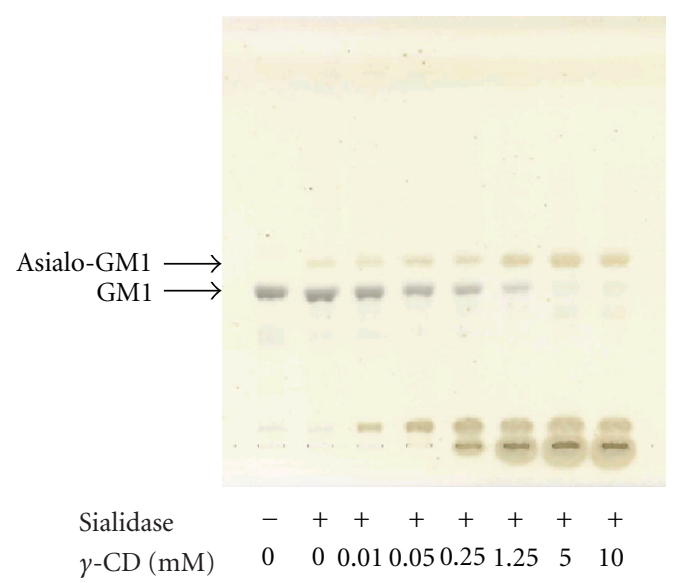

(a)

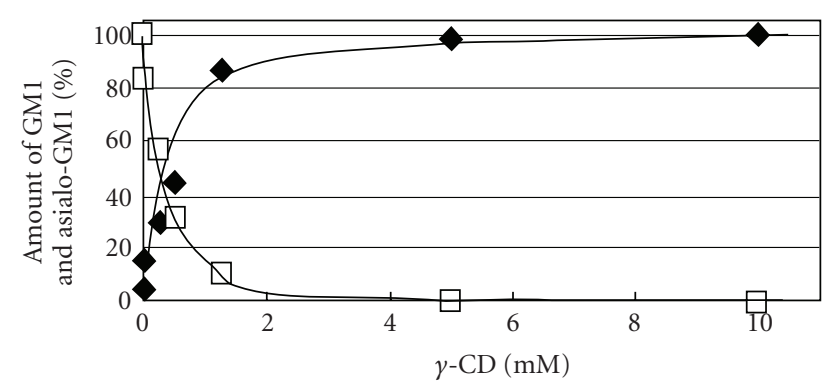

(b)

Figure 2: Effect of the concentration of $\gamma$-CD. $200 \mathrm{mU}$ of sialidase was added $(+)$, or not added $(-)$ to the reaction solution. HPTLC analysis was performed as described in Figure 1. (a) Result of HPTLC analysis. (b) The amounts of ganglioside GM1 and asialoGM1 after enzymatic reaction. $\square$ : ganglioside GM1; : asialo-GM1.

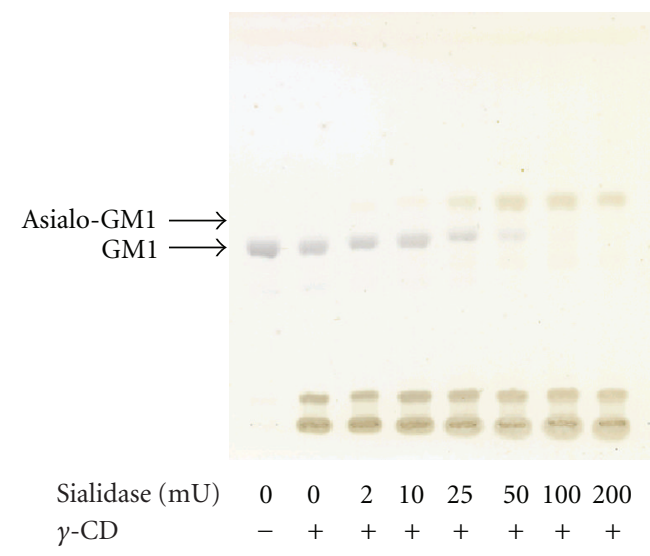

(a)

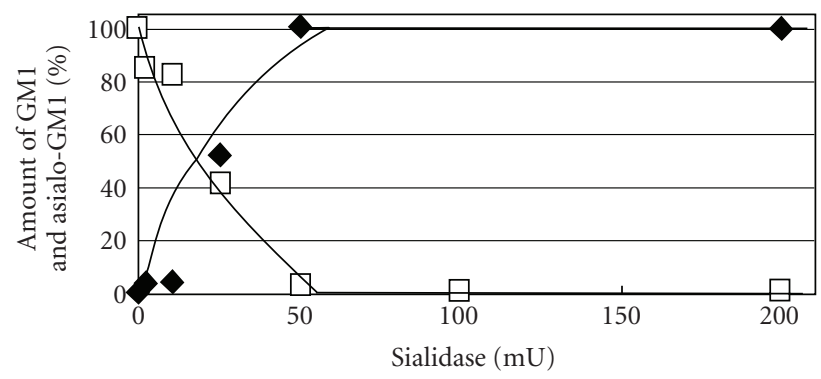

(b)

FIGURE 3: Effect of the concentration of sialidase. $5 \mathrm{mM}$ of $\gamma$-CD was added to reaction solution $(+)$, or not added $(-)$. HPTLC analysis was performed as described in Figure 1. (a) Result of HPTLC analysis. (b) The amounts of ganglioside GM1 and asialo-GM1 after enzymatic reaction. $\square$ : ganglioside GM1; $\diamond$ : asialo-GM1. 
2.2. Sialidase Reaction. The reaction mixture for sialidase was comprised of $3 \mu \mathrm{g}$ of ganglioside GM1 (or $10 \mu \mathrm{g}$ of crude ganglioside mixture), $0.1 \mathrm{M}$ acetate buffer, $\mathrm{pH}$ 5.5, and $2-500 \mathrm{mU}$ of $A$. ureafaciens sialidase, to a final volume of $200 \mu \mathrm{L}$. Then the solution was incubated at $37^{\circ} \mathrm{C}$ for 24 hours [16]. After the reaction, the solution was treated with a SepPak C-18 cartridge immediately [17]. The entire sample was analyzed by high-performance thin-layer chromatography (HPTLC). The addition of other components is mentioned in the text.

2.3. HPTLC Analysis. Amphiphilic glycolipids were analyzed on an HPTLC plate (Merck, Darmstadt, Germany). The developing solvent used was chloroform-methanol- $0.25 \%$ aqueous $\mathrm{KCl}(5: 4: 1, \mathrm{v} / \mathrm{v})$. The separated glycolipids were detected by spraying with resorcinol reagent and heating at $120^{\circ} \mathrm{C}$ for 15 minutes in an oven $[17,18]$. Densitogram was obtained from HPTLC by using Scion Image Software (Scion Corporation, http://www.scioncorp.com) to quantify glycolipids.

2.4. Mass Spectral Analysis. Mass spectra were recorded on a high-capacity ion trap mass spectrometer (HCTultra). At least ten scans were averaged to obtain each spectrum. Samples were typically dissolved in chloroform/methanol $(2: 1, \mathrm{v} / \mathrm{v})$ to a concentration of $1 \mathrm{mg} / \mathrm{mL}$, and diluted to $1 / 1000$ with $95 \%$ acetonitrile. Sample solution was injected to the ESI ion source by mechanical infusion at a flow rate of $180 \mu \mathrm{L} / \mathrm{h}$. Analysis of neutral glycolipids was carried out in positive ion mode. The following analysis parameters were used: high voltage $(\mathrm{HV})$ capillary voltage $-4500 \mathrm{~V}$, $\mathrm{HV}$ end plate offset voltage $-500 \mathrm{~V}$, nebulizer gas pressure $15.0 \mathrm{psi}$ (nitrogen), a flow rate of dry gas $7.0 \mathrm{l} / \mathrm{min}$ (nitrogen, temperature $320^{\circ} \mathrm{C}$ ), and skimmer voltage $40.0 \mathrm{~V}$.

\section{Results and Discussion}

We attempted to prepare asialo gangliosides by using $A$. ureafaciens sialidase (mixture of isoenzymes L, M1, M2, and S) and sodium cholate. $10 \mu \mathrm{g}$ of crude ganglioside mixture (from rat brain) was hydrolyzed under various conditions and the purified sample was analyzed by HPTLC. The addition of sodium cholate into the reaction solution was effective for the cleavage of the internal sialic acid residue of GM1. However, sodium cholate could not be removed by using a Sep-Pak C18 cartridge and impeded HPTLC analysis (Figure 1(a)). Generally, removal of detergents such as sodium cholate, sodium deoxycholate, and sodium chenodeoxycholate is complicated. Recently, it was reported that $\gamma$-CD was an efficient supporting material for the blotting of hydrophobic substrates to water-soluble polymers and for the synthesis of neoglycolipids using glycosyltransferase [13]. Therefore, a stimulating effect of $\gamma$-CD on sialidase reaction was investigated. As shown in Figure 1(b), cleavage of the internal sialic acid residue of GM1 was effectively promoted by $\gamma$-CD and asialo-GM1 could be completely separated from $\gamma$-CD by HPTLC. $\gamma$-CD has the ability to include the hydrophobic part of compounds and the inclusion

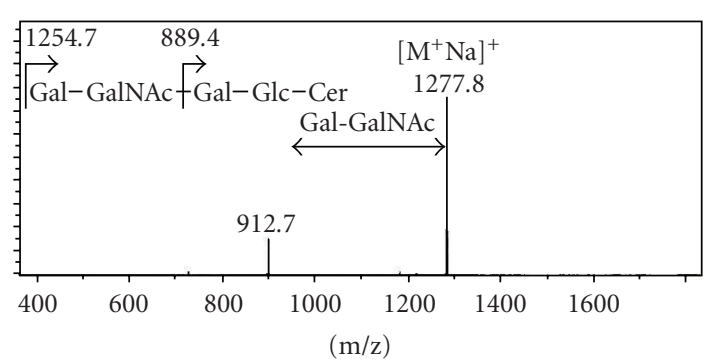

FIGURE 4: Mass Spectra of the product from ganglioside GM1 after sialydase reaction. Analysis of sample was carried out on highcapacity ion trap mass spectrometer (HCTultra) in positive mode. The product was prepared by scraping off the adsorbent at the corresponding spot site on the HPTLC plate.

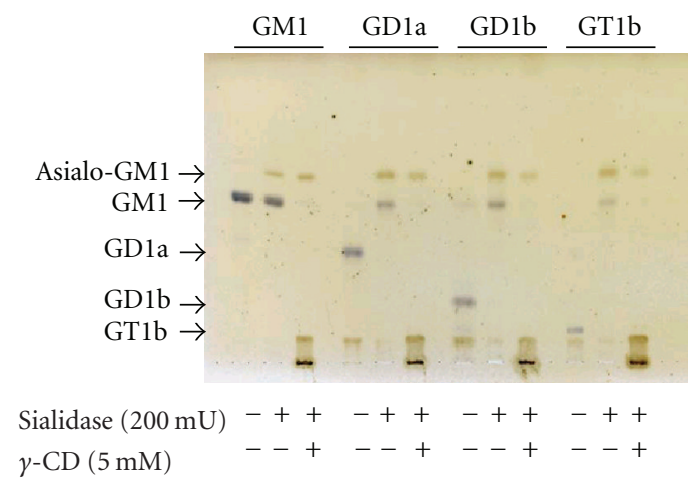

FIgURE 5: Desialylation of various kinds of ganglioside. HPTLC analysis was performed as described in Figure 1.

complex becomes more soluble in the reaction solvent when compared with the noncomplexed compounds. It has been reported that $\alpha$-CD and dimethyl- $\beta$-cyclodextrin activate the hydrolysis of ganglioside GM1 by acid $\beta$-galactosidases [19]. The activation of the hydrolysis by sialidase may correlate with the inclusion complex formation according to a mechanism suggested for the acid $\beta$-galactosidases reactions.

Todetermine the optimum $\gamma$-CD concentration for sialidase reaction, $\gamma$-CD was added to the reaction solution at a final concentration of $0.01,0.05,0.25,1.25,5$, or $10 \mathrm{mM}$. An increase of the concentration of $\gamma$-CD allows for ganglioside GM1 to be converted to asialo-GM1 (Figure 2). GM1 completely hydrolyzed at concentrations of $\gamma$-CD higher than $5 \mathrm{mM}$. $\alpha$-CD and $\beta$-CD were not as efficiently enhancing cleavage of the internal sialic acid as $\gamma$-CD at a final concentration of $5 \mathrm{mM}$. The yields of asialo-GM1 produced in the presence of $\alpha-\mathrm{CD}$ and $\beta$-CD were 3 and $10 \%$ of the yield of that produced in the presence of $\gamma$ $\mathrm{CD}$, respectively. In the presence of $5 \mathrm{mM}$ of $\gamma$-CD, $3 \mu \mathrm{g}$ ganglioside GM1 has been completely digested by $100 \mathrm{mU}$ of A. ureafaciens sialidase (Figure 3 ). The product of enzymecatalyzed desialylation from ganglioside GM1 was identified by MS/MS analyses. As shown in Figure 4, the parent and 
fragment mass ions of asialo-GM1 were detected as monovalent cations $\left(\mathrm{m} / \mathrm{z}\right.$ 1277.8: $[\mathrm{M}+\mathrm{Na}]^{+}$and $\mathrm{m} / \mathrm{z}$ 912.7: $[\mathrm{M}-$ Gal-GalNAc+Na $]^{+}$). Under optimum condition described above, various kinds of gangliosides (GM1, GD1a, GD1b, and DT1b) were completely deacylated by $A$. ureafaciens sialidase (Figure 5).

$\gamma$-CD does not disturb HPTLC and HPLC analysis and is useful for enzymatic desialylation. Nagashima et al. have reported that $\gamma$-CD is an efficient supporting material for the blotting of hydrophobic substrate and for the synthesis of neoglycolipids using glycosyltransferase [13]. The utility of $\gamma$-CD is an efficient and widely useful method for chemical and enzymatic reactions of glycolipids and (oligo)saccharide derivatives. Furthermore, asialo glycolipid will be useful for study of a physiological function and the industrial use by effective preparation methods.

\section{Acknowledgments}

This work was supported by a grant for "Development of Novel Diagnostic and Medical Applications through Elucidation of Sugar Chain Functions" from New Energy and Industrial Technology Development Organization (NEDO). The authors thank Dr. H. Shimizu (National Institute of Advanced Industrial Science and Technology, Hokkaido Center, Sapporo, Japan) for his helpful comments and suggestions on this study. The first two authors equally contributed to this work.

\section{References}

[1] Y. Nagai and M. Iwamori, "Cellular biology of gangliosides," in Biology of the Sialic Acids, A. Rosenberg, Ed., pp. 197-241, Plenum Press, New York, NY, USA, 1995.

[2] S.-I. Hakomori, "Cancer-associated glycosphingolipid antigens: their structure, organization, and function," Acta Anatomica, vol. 161, no. 1-4, pp. 79-90, 1998.

[3] A. Varki, "Biological roles of oligosaccharides: all of the theories are correct," Glycobiology, vol. 3, no. 2, pp. 97-130, 1993.

[4] M. Saito and R. K. Yu, "Biochemisty and function of sialidases," in Biology of the Sialic Acids, A. Rosenberg, Ed., pp. 261-313, Plenum Press, New York, NY, USA, 1995.

[5] R. Schauer and J. G. Vliegenthart, "Introduction to sialic acids," in Sialic Acids: Chemistry, Metabolism and Functions, R. Schauer, Ed., pp. 1-3, Springer, Vienna, Austria, 1982.

[6] M.-Y. Chou, S.-C. Li, M. Kiso, A. Hasegawa, and Y.-T. Li, "Purification and characterization of sialidase L, a NeuAc $\alpha 2 \rightarrow$ 3Gal-specific sialidase," The Journal of Biological Chemistry, vol. 269, no. 29, pp. 18821-18826, 1994.

[7] M.-Y. Chou, S.-C. Li, and Y.-T. Li, "Cloning and expression of sialidase L, a NeuAc $\alpha 2 \rightarrow 3 \mathrm{Gal}$-specific sialidase from the leech, Macrobdella decora," The Journal of Biological Chemistry, vol. 271, no. 32, pp. 19219-19224, 1996.

[8] T. Corfield, "Bacterial sialidases—roles in pathogenicity and nutrition," Glycobiology, vol. 2, no. 6, pp. 509-521, 1992.

[9] M. Iwamori, Y. Ohta, Y. Uchida, and Y. Tsukada, "Arthrobacter ureafaciens sialidase isoenzymes, L, $\mathrm{m} 1$ and $\mathrm{m} 2$, cleave fucosyl GM1," Glycoconjugate Journal, vol. 14, no. 1, pp. 67-73, 1997.

[10] M. Iwamori, S. Sunada, E. Ishihara, M. Moki, S. Fujimoto, and Y. Nagai, "Differential expression of fucosyl GM1 and a disialoganglioside with a NeuAc $\alpha 2-6 \mathrm{Ga} 1 \mathrm{NAc}$ linkage (GD1e) in various rat ascites hepatoma cells," FEBS Letters, vol. 198, no. 1, pp. 66-70, 1986.

[11] L. O. Sillerud, R. K. Yu, and D. E. Schafer, "Assignment of the carbon-13 nuclear magnetic resonance spectra of gangliosides GM4, GM3, GM2, GM1, GD1a, GD1b, and GT1b," Biochemistry, vol. 21, no. 6, pp. 1260-1271, 1982.

[12] M. Iwamori, T. Kaido, Y. Iwamori, Y. Ohta, K. Tsukamoto, and S. Kozaki, "Involvement of the C-terminal tail of Arthrobacter ureafaciens sialidase isoenzyme $\mathrm{M}$ in cleavage of the internal sialic acid of ganglioside GM1," The Journal of Biochemistry, vol. 138, no. 3, pp. 327-334, 2005.

[13] I. Nagashima, H. Shimizu, T. Matsushita, and S.-I. Nishimura, "Chemical and enzymatic synthesis of neoglycolipids in the presence of cyclodextrins," Tetrahedron Letters, vol. 49, no. 21, pp. 3413-3418, 2008.

[14] H. Tsuda, K. Sekine, N. Takasuka, H. Toriyama-Baba, and M. Iigo, "Prevention of colon carcinogenesis and carcinoma metastasis by orally administered bovine lactoferrin in animals," BioFactors, vol. 12, no. 1-4, pp. 83-88, 2000.

[15] B. Gatterbauer, A. Neisser, H. Bernheimer, and B. Schwerer, "Antiglycosphingolipid immune responses in neurology: the Vienna experience with isotypes, subclasses, and disease," Annals of the New York Academy of Sciences, vol. 845, pp. 353362, 1998.

[16] Y. Uchida, Y. Tsukada, and T. Sugimori, "Enzymatic properties of neuraminidases from Arthrobacter ureafaciens," The Journal of Biochemistry, vol. 86, no. 5, pp. 1573-1585, 1979.

[17] M. C. Z. Kasuya, L. X. Wang, Y. C. Lee, et al., "Azido glycoside primer: a versatile building block for the biocombinatorial synthesis of glycosphingolipid analogues," Carbohydrate Research, vol. 329, no. 4, pp. 755-763, 2000.

[18] L. Svennerholm, "Quantitive estimation of sialic acids-II: a colorimetric resorcinol-hydrochloric acid method," Biochimica et Biophysica Acta, vol. 24, pp. 604-611, 1957.

[19] T. Shiraishi, M. Hiraiwa, and Y. Uda, "Effects of cyclodextrins on the hydrolysis of ganglioside $\mathrm{G}_{\mathrm{M} 1}$ by acid $\beta$-galactosidases," Glycoconjugate Journal, vol. 10, no. 2, pp. 170-174, 1993. 


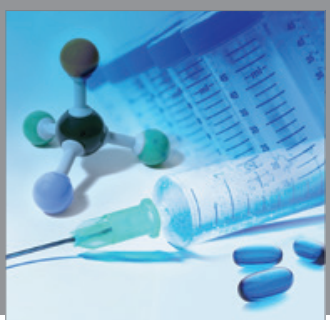

International Journal of

Medicinal Chemistry

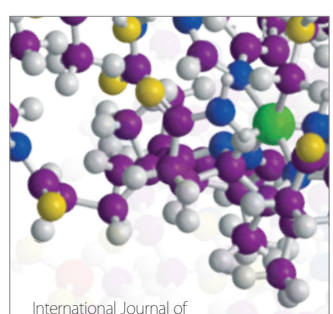

Carbohydrate Chemistry

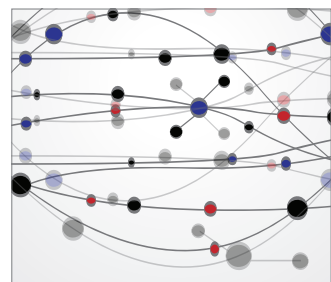

The Scientific World Journal
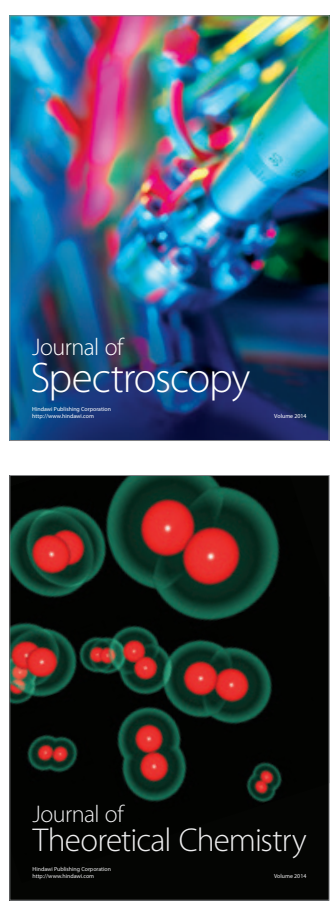
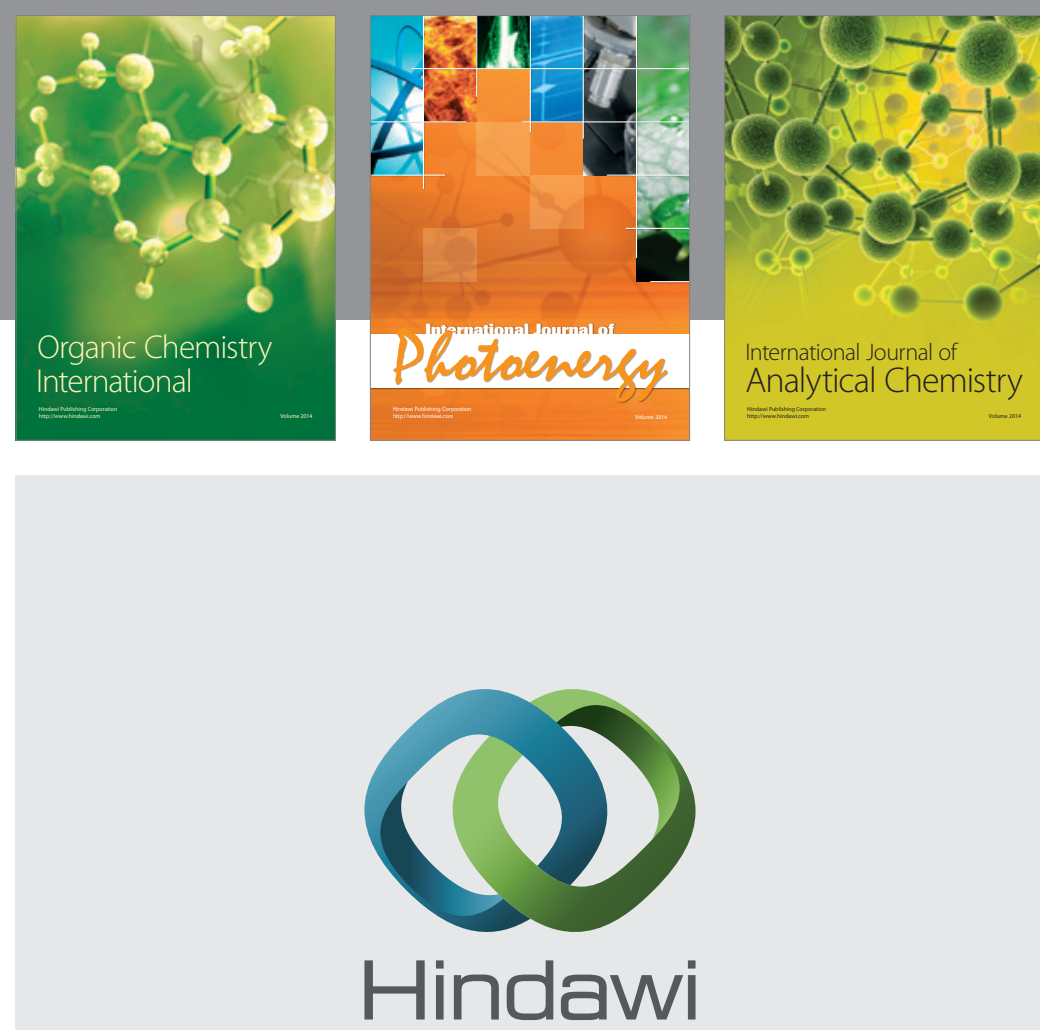

Submit your manuscripts at

http://www.hindawi.com
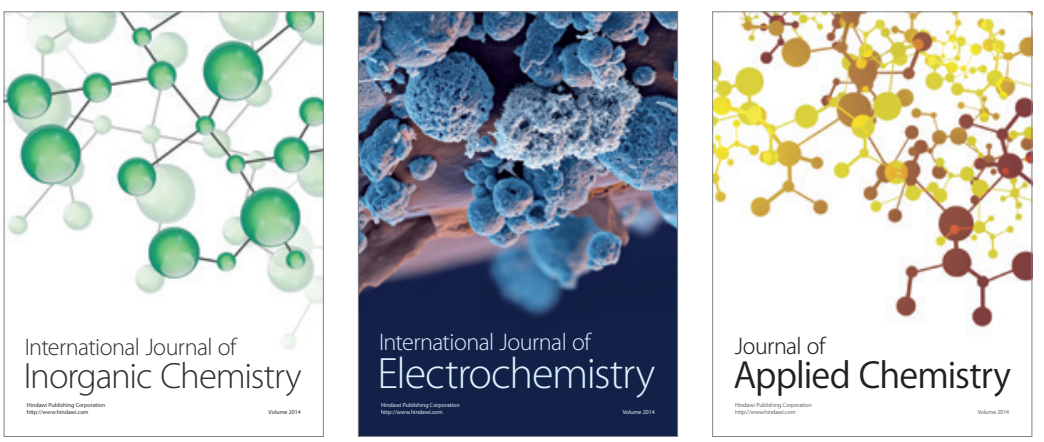

Journal of

Applied Chemistry
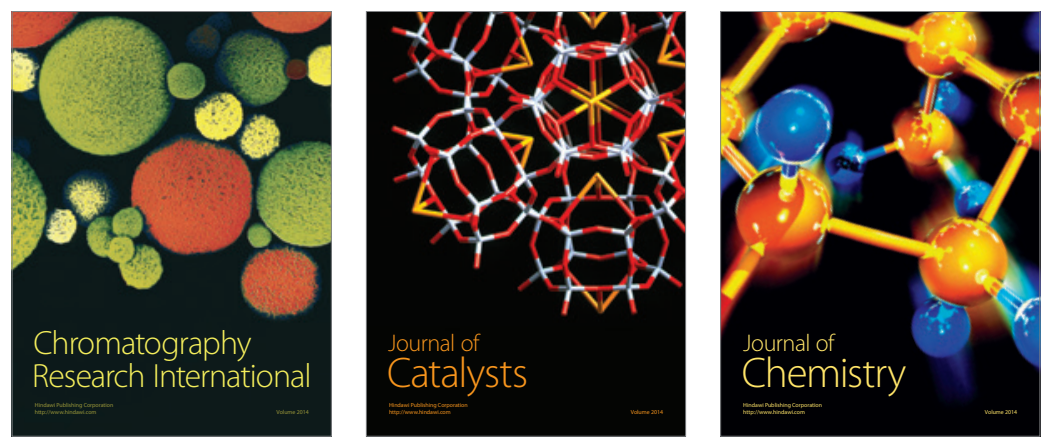
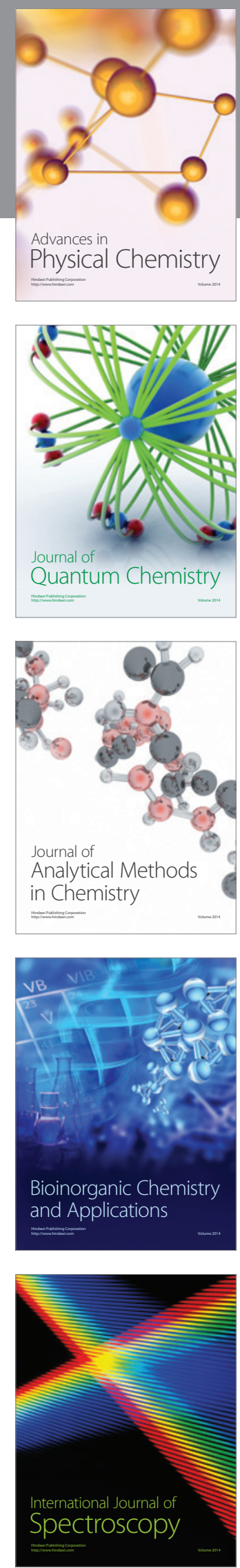